\title{
Penetração intratubular de cimentos endodônticos
}

\section{Intratubular penetration of root canal sealers}

\author{
Gustavo de Deus* \\ Eduardo Diogo Gurgel Filho** \\ Cláudio Maniglia Ferreira** \\ Tauby Coutinho Filho*
}

\begin{abstract}
RESUMO: Este estudo foi desenvolvido para avaliar a capacidade de penetração de diferentes cimentos endodônticos (Endo Fill, Sealapex, AH Plus e Pulp Canal Sealer) nos túbulos dentinários em dentes devidamente modelados e obturados. Foram utilizados 72 incisivos centrais superiores, os quais foram instrumentados no sentido coroa-ápice pela técnica de forças balanceadas. O comprimento de trabalho foi estabelecido a $1 \mathrm{~mm}$ do ápice radicular. Durante a limpeza e modelagem, todos os dentes foram irrigados com $10 \mathrm{ml}$ de hipoclorito de sódio a 5,25\%. Os dentes foram divididos em 4 grupos, sendo 1 para cada tipo de cimento. Estes grupos foram então subdivididos em função do uso ou não de EDTA a 17\% previamente à obturação dos canais radiculares, para a remoção da lama dentinária. Todos os dentes foram obturados pela técnica da onda de condensação com cone médio calibrado. Após obturação, as raízes foram seccionadas no sentido mésio-distal e foi escolhida a secção de melhor qualidade visual. Estas foram então analisadas em microscopia eletrônica de varredura (MEV), sendo o foco de observação sempre a interface dentina/material obturador. Após obtenção das imagens, mensurou-se os prolongamentos dos cimentos para o interior dos túbulos dentinários. O cimento de Rickert (Pulp Canal Sealer) apresentou a maior capacidade de penetração nos túbulos dentinários, sendo os piores resultados apresentados pelo grupo em que se utilizou o Sealapex. Os resultados foram avaliados estatisticamente pelo teste de Spearman, o qual mostrou diferença estatisticamente significante $(p \leq 0,01)$ entre os grupos em que o EDTA foi utilizado.
\end{abstract}

DESCRITORES: Obturação do canal radicular; Cimentos dentários; Túbulos dentinários.

\begin{abstract}
The purpose of this study was to evaluate the capacity of penetration of four endodontic sealers (Endo Fill, Sealapex, AH Plus and Pulp Canal Sealer) into dentinal tubules. Seventy-two extracted human maxillary anterior teeth were utilized in this study. The teeth were cleaned and shaped by means of the balanced-forces technique. The work length was established at $1 \mathrm{~mm}$ beyond the apex. Copious irrigation with $10 \mathrm{ml}$ of $5.25 \%$ sodium hypochlorite was carried out. The teeth were divided in 8 groups -4 had the smear layer maintained, and 4 had it removed. The smear layer was removed with a commercial solution of $17 \%$ EDTA, and the root canal system was flushed for 3 min. Finally, the roots were irrigated with $3 \mathrm{ml}$ of $5.25 \%$ sodium hypochlorite. All teeth were sealed by means of the technique of the condensation wave with a medium nonstandardized cone. After filling, the roots were grooved, longitudinally split and examined under a scanning electron microscope (SEM). The focus of observation was the interface between the dentin and the sealing material. The Rickert sealer (Pulp Canal) presented the maximum penetration depths into the dentinal tubules, and Sealapex, the minimum. The Spearman test was used to determine whether there were significant differences between the groups. The removal of smear layer allowed significant penetration of the sealers ( $\mathrm{p} \leq 0.01)$.
\end{abstract}

DESCRIPTORS: Root canal obturation; Dental cements; Dentinal tubules.

\section{INTRODUÇÃO}

As técnicas modernas de obturação procuram lançar mão de uma maior quantidade de guta-percha e de uma menor película de cimento, visto que hoje tem-se a consciência de que o cimento representa a porção frágil da obturação ${ }^{8,9}$. Ainda assim, os cimentos endodônticos continuam representando um importante papel no controle da percolação apical, escoando para as ramificações e melhorando a adaptação da obturação nas irregularidades da interface dentina-material obturador.
Devido a estes aspectos, os cimentos endodônticos despertam o interesse dos pesquisadores ${ }^{4,6}$.

É descrito que a tendência futura em relação às substâncias cimentantes deve idealizar que estes materiais ocupem os túbulos dentinários, unamse intimamente às fases orgânicas e inorgânicas da dentina, destruam ou neutralizem microrganismos e seus subprodutos, induzam a neoformação cementária e fortaleçam o sistema de canais radiculares. Devemos frisar, que dentro desta perspec-

*Professores da Disciplina de Endodontia - Universidade do Estado do Rio de Janeiro.

**Professores da Disciplina de Endodontia - Universidade de Fortaleza. 
de Deus G, Gurgel Filho ED, Ferreira CM, Coutinho Filho T. Penetração intratubular de cimentos endodônticos. Pesqui Odontol Bras 2002;16(4):332-336.

tiva, todos os cimentos utilizados atualmente podem ser considerados inadequados ${ }^{3}$.

O objetivo deste trabalho foi analisar a profundidade de penetração intradentinária de quatro cimentos endodônticos, quando empregados na técnica de obturação pela onda de condensação ${ }^{1}$.

\section{MATERIAL E MÉTODOS}

No presente trabalho, foram selecionados 72 incisivos centrais superiores humanos do banco de dentes da FO-UERJ, medindo entre $22 \mathrm{~mm}$ e $26 \mathrm{~mm}$ de comprimento, todos apresentando canais amplos e forames patentes.

As amostras foram todas acessadas de maneira convencional e preparadas no sentido coroa-ápice, utilizando-se brocas de Gates-Glidden (Dentsply Maillefer Instruments SA, Ballaigues, Suíça) números 5, 4 e 3, no sentido decrescente. O preparo manual foi feito com limas tipo K (Dentsply - Maillefer Instruments SA, Ballaigues, Suíça) pela técnica de forças balanceadas ${ }^{2}$, sendo o comprimento de trabalho estabelecido a $1 \mathrm{~mm}$ do ápice e o batente apical confeccionado com lima número 50. Foram utilizados $10 \mathrm{ml}$ de hipoclorito de sódio a 5,25\% (Biodinâmica Química e Farmacêutica Ltda., Ibiporã, PR, Brasil) durante a instrumentação.

Após a fase de instrumentação os elementos foram divididos aleatoriamente em quatro grupos de dezoito elementos cada: A, B, C e D. Posteriormente subdivididos em subgrupos: A1/A2, B1/B2, $\mathrm{C} 1 / \mathrm{C} 2$ e D1/D2, com nove elementos cada. Os subgrupos A1, B1, C1 e D1 foram irrigados apenas com hipoclorito de sódio a 5,25\%. Nos subgrupos A2, B2, C2 e D2 foi realizado o toalete final utilizando uma solução de EDTA 17\% (Biodinâmica Química e Farmacêutica Ltda., Ibiporã, PR, Brasil) agitados mecanicamente por 3 minutos. Em seguida foi realizada uma última lavagem com $1 \mathrm{ml} \mathrm{de}$ hipoclorito de sódio a 5,25\%.

Os canais foram secos e obturados com cones acessórios médios (DiaDent Group International Inc., Seul, Coréia) utilizados como cones principais pela técnica da onda de condensação ${ }^{1}$ utilizando o aparelho System B (Analytic Technology, Redmond, EUA).

As pontas destes cones foram calibradas no diâmetro $0,50 \mathrm{~mm}$ com auxílio de régua de calibração (Dentsply - Maillefer Instruments SA, Ballaigues, Suiça), que corresponde à última lima utilizada no comprimento de trabalho, para a formação do batente apical. A ponta média do apare- lho System B, bem como o calcador de Schilder (JR Instrumentos Ltda., BH, Brasil), foram selecionados e um cursor foi colocado determinando o limite de penetração da ponta do System B a $5 \mathrm{~mm}$ aquém do comprimento de trabalho. Estando a temperatura ajustada em $200^{\circ} \mathrm{C}$ foi feita a onda de condensação até o comprimento preestabelecido. Neste momento interrompeu-se o aquecimento da guta-percha por 10 segundos. Um novo acionamento de 5 segundos é feito para remoção do excesso de guta-percha e manutenção do terço apical obturado que deve ser condensado. A obturação dos terços cervical e médio foi feita através do aparelho Obtura II (Obtura Corp., Fenton, MO, EUA) com incrementos de $3 \mathrm{~mm}$ até a entrada do canal.

No grupo A foi utilizado o cimento de Grossman (Endo Fill, Dentsply Indústria e Comércio Ltda., Brasil), no grupo B o AH Plus (Dentsply DeTrey Gmbh, Alemanha), no grupo $\mathrm{C}$ o Sealapex (Kerr Corporation, Orange, EUA) e no grupo D, a fórmula original de Rickert (Pulp Canal Sealer, Kerr Corporation, Orange, EUA). Todos os cimentos foram espatulados seguindo as orientações dos fabricantes. Para cada amostra obturada foi utilizado uma porção predeterminada de cimento, equivalente a $1,25 \mathrm{ml}$.

Os dentes foram mantidos em ambiente com $100 \%$ de umidade por duas semanas. Após esse período, as raízes foram seccionadas no sentido mésio-distal (Figura 1) e a secção de melhor qualidade visual foi escolhida para a análise. Estas foram então colocadas em placas de alumínio, codificadas e devidamente preparadas para análise ao microscópio eletrônico de varredura (MEV).

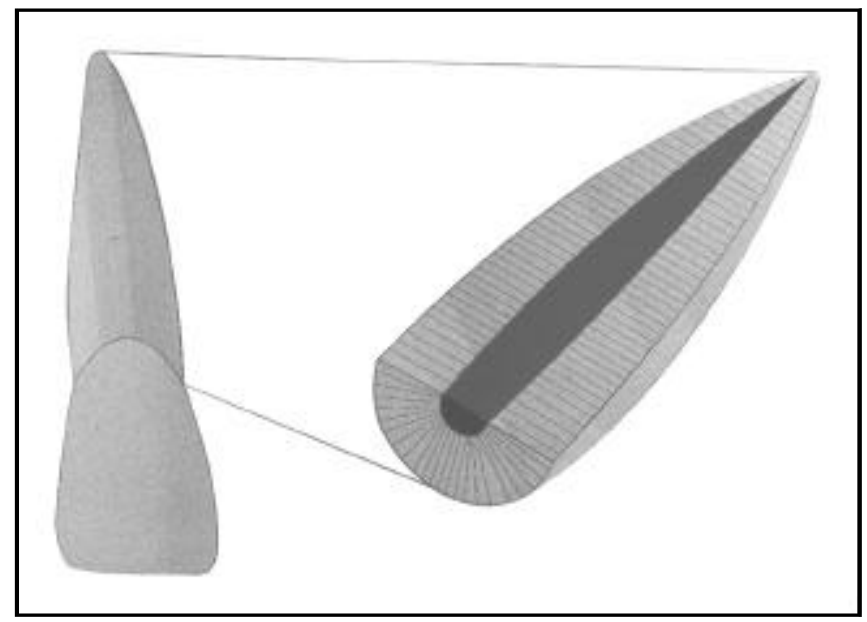

FIGURA 1 - Esquema demonstrativo da clivagem realizada nas amostras. 
de Deus G, Gurgel Filho ED, Ferreira CM, Coutinho Filho T. Penetração intratubular de cimentos endodônticos. Pesqui Odontol Bras 2002;16(4):332-336.

De cada amostra foram obtidas quatro fotomicrografias da região média, exatamente a $8 \mathrm{~mm}$ do ápice, com aumentos que variavam entre 150 a $10.000 \mathrm{X}$, sendo o foco de observação sempre a interface dentina/material obturador. Utilizando os recursos presentes no próprio $\mathrm{MEV}$, foi realizada a mensuração dos picos que representavam as menores e maiores profundidades de penetração do cimento endodôntico.

As medidas encontradas foram arquivadas em uma planilha eletrônica e tratadas estatisticamente através do teste de Spearman.

\section{RESULTADOS}

A penetração intradentinária dos cimentos endodônticos não foi considerada significante em nenhuma das amostras em que o EDTA não foi

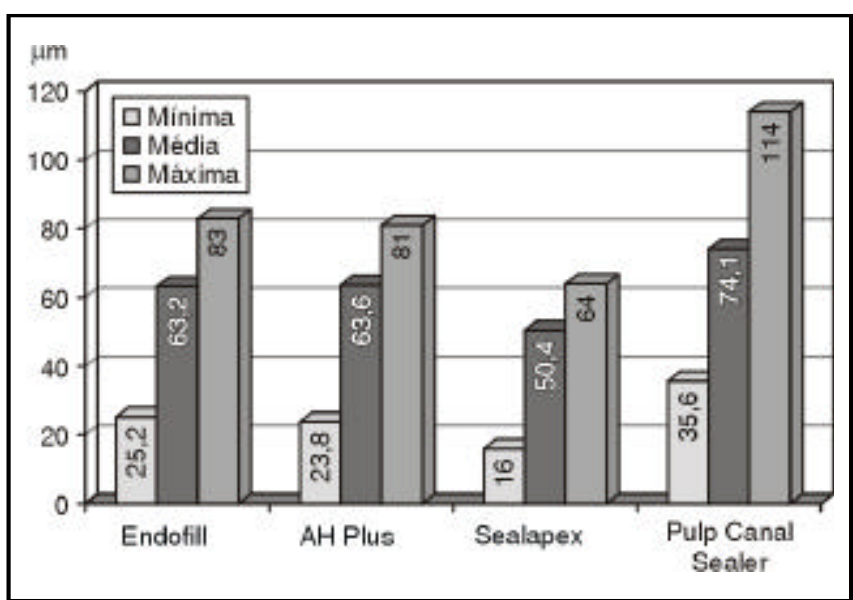

GRÁFICO 1 - Valores das penetrações mínimas, médias e máximas de cada grupo.

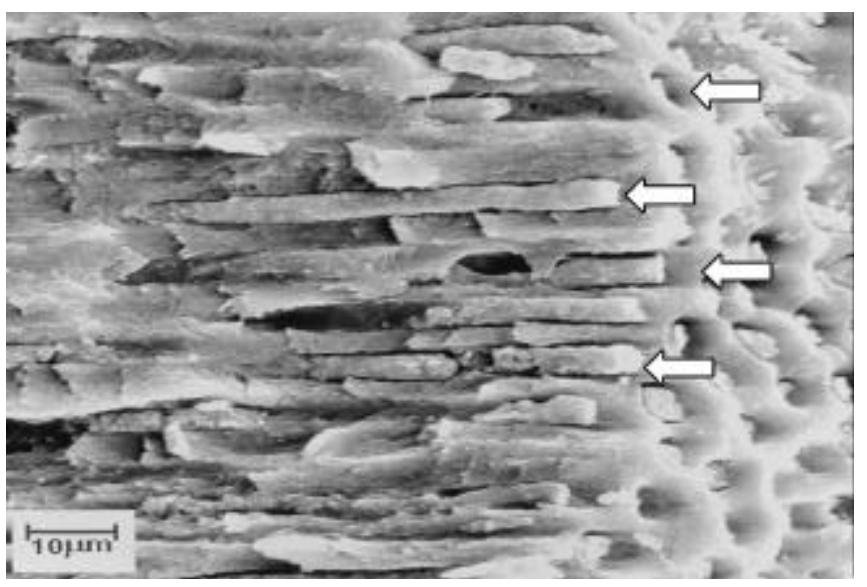

FIGURA 2 - Evidenciação da penetração do Pulp Canal Sealer (setas) em vários túbulos dentinários. Grupo D2 (1.000 X). utilizado (subgrupos controles). Essa condição confirma a validade na remoção da lama dentinária.

De um modo geral, a penetração dos cimentos nos túbulos dentinários foi facilmente observada nos subgrupos experimentais.

Os postos médios, bem como os valores máximos e mínimos da profundidade de penetração dos cimentos, encontram-se expressos no Gráfico 1. A análise estatística demonstrou haver diferença significante entre os grupos D2 (cimento de Rickert - Pulp Canal Sealer) e C2 (Sealapex), para $\mathrm{p} \leq 0,01$. Para os demais grupos, não ficou constatada diferenças estatisticamente significantes.

As penetrações dos diferentes cimentos endodônticos nos túbulos dentinários estão exemplificadas nas Figuras 2 a 7 .

\section{DISCUSSÃO}

Objetivando uma melhor qualidade na adaptação do material obturador às paredes do sistema de canais radiculares, os cimentos endodônticos vêm sendo empregados como coadjuvantes da obturação ${ }^{9}$. Está presente na literatura o constante interesse em novos materiais para atuarem como selantes tais como, os cimentos de policarboxilato, cianoacrilato, cimento de ionômero de vidro e adesivos dentinários, os quais revelaram uma grande dificuldade de manipulação clínica, gerando sensibilidade de técnica ${ }^{6,12}$.

Quando utilizamos técnicas de obturação termoplásticas, temos a consciência que a quantidade de cimento utilizada diminui ${ }^{11}$. Porém essa pequena película de cimento deverá estar bem adaptada às

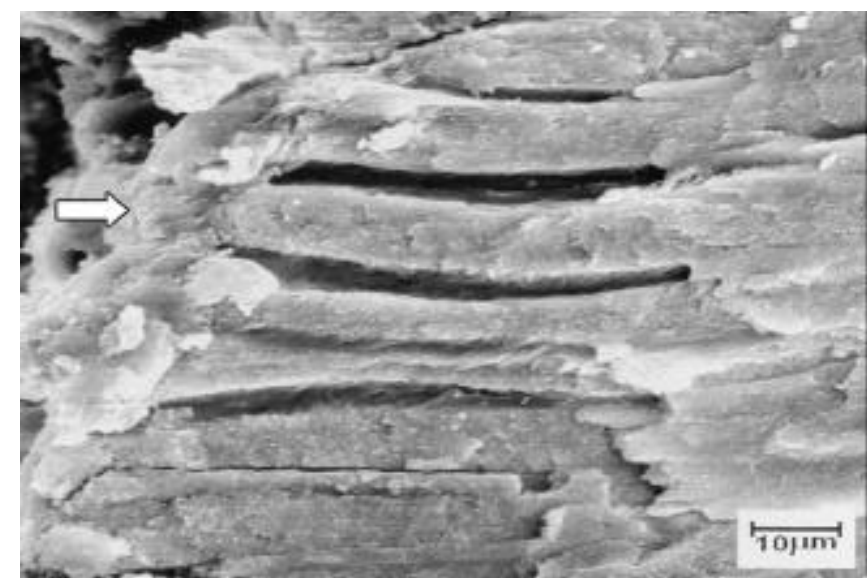

FIGURA 3 - Lama dentinária (seta) impedindo a penetração do cimento Endofill nos túbulos dentinários. Grupo A1 (500 X). 


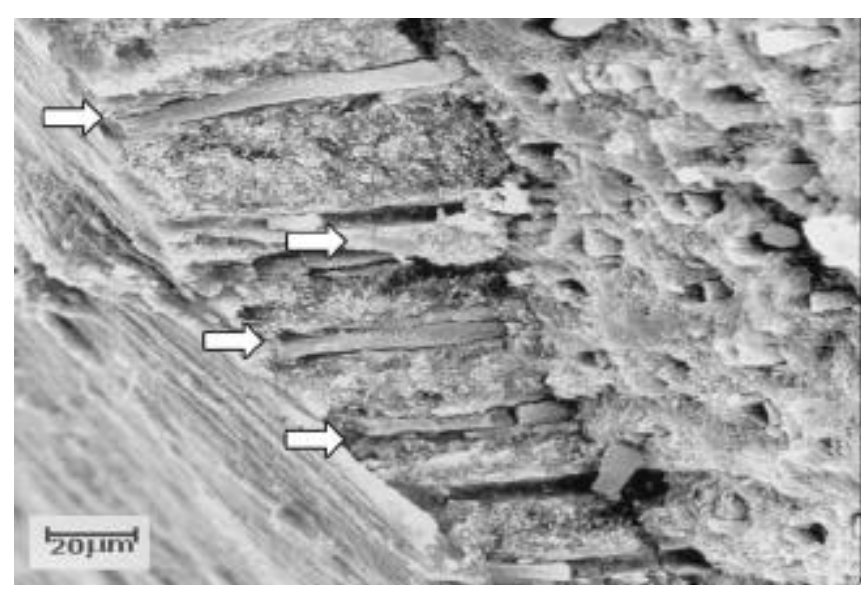

FIGURA 4 - Cimento AH Plus com cerca de $85 \mu \mathrm{m}$ de profundidade de penetração. Grupo B2 (500 X).

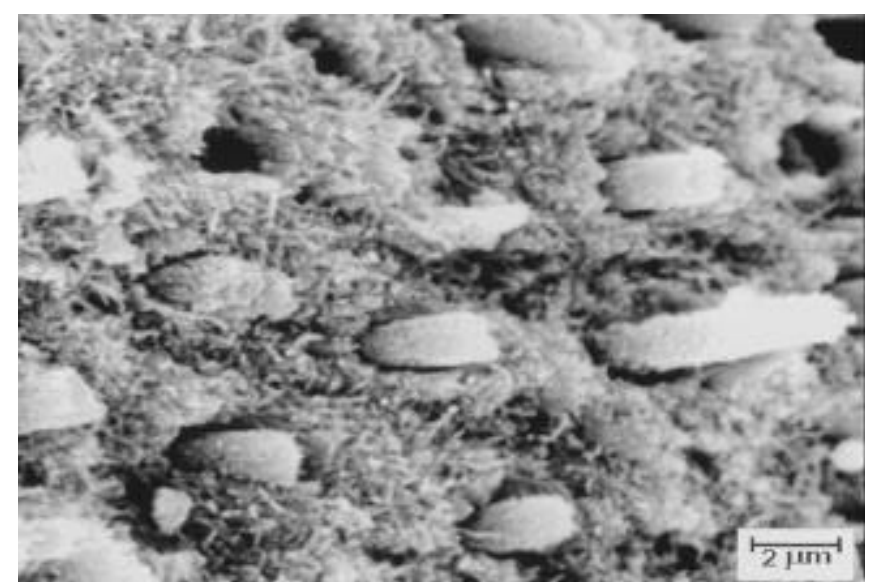

FIGURA 6 - Aspecto do cimento Sealapex saindo dos túbulos dentinários. Grupo C2 (2.000 X).

paredes dentinárias, tendo, se possivel penetrado nos túbulos dentinários ${ }^{4}$.

Essa adaptação vem sendo analisada por diversas pesquisas e a microscopia eletrônica de varredura (MEV) vem sendo o método escolhido para a observação dessa penetração ${ }^{4}$. Através da imagem formada pelo feixe de elétrons do MEV, pode-se observar o escoamento do cimento nos túbulos dentinários patentes ${ }^{5,7}$. Através desta metodologia, toda a superficie da parede dentinária pode então ser examinada e caracterizada com riqueza de detalhes.

Os nossos resultados mostraram uma melhor capacidade de penetração intradentinária do cimento de Rickert, o que pode justificar os resultados obtidos por Yared, Bou-Dagher ${ }^{11}$ (1996), que observaram menor infiltração no cimento Pulp Canal Sealer (Rickert) quando comparado ao cimento de Grossman e ao AH26.

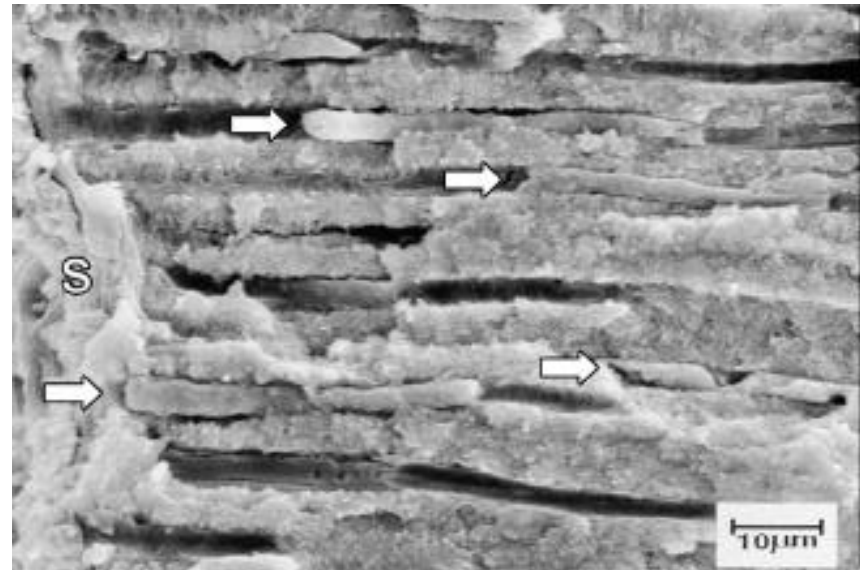

FIGURA 5 - Camada de cimento Pulp Canal Sealer (S) e seu prolongamento (setas) dentro dos túbulos dentinários. Grupo D2 (1.000 X).

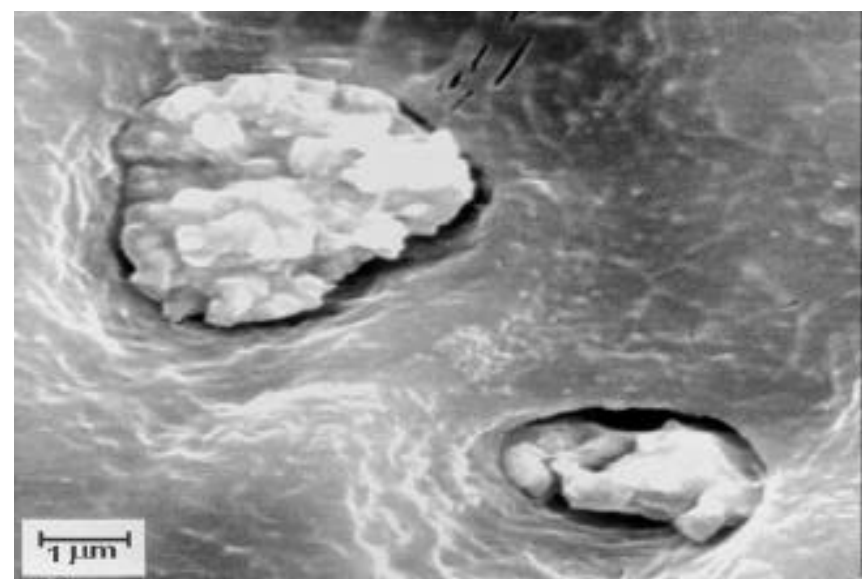

FIGURA 7 - Evidenciação do aspecto microparticulado do cimento Pulp Canal Sealer. Grupo D2 (10.000 X).

Os resultados da penetração encontrados no trabalho de Kouvas et al. ${ }^{4}$ (1998) estão numericamente de acordo com os da nossa pesquisa e com os relatados por Oksan et al. ${ }^{6}$ (1993). Porém, Sem et $a .^{9}$ (1996) obtiveram medidas variando de 60 a $800 \mu \mathrm{m}$. Essa grande diferença de valores pode ser devido a algum desvio de metodologia entre essas pesquisas, principalmente quando consideramos a dificuldade de obtermos uma clivagem apropriada, que nos forneça amostragens mais homogêneas.

Nossos resultados mostraram diferentes profundidades de penetração intradentinária entre os diferentes cimentos endodônticos testados, fato explicado por Oksan et al. ${ }^{6}$ (1993) que relataram que a composição química do cimento e suas características físicas, tais como: a capacidade de escoamento, viscosidade e os tamanhos das par- 
tículas devam exercer influência significante na capacidade destas substâncias penetrarem no tecido dentinário.

Os piores resultados obtidos foram para o grupo do Sealapex, justificado pela baixa capacidade de escoamento deste cimento ${ }^{10}$.

Os resultados obtidos nos quatro subgrupos em que o EDTA não foi utilizado, evidenciaram a influência negativa que a lama dentinária exerce sobre a capacidade de penetração intradentinária dos cimentos endodônticos, observação também citada por Leonard et al. ${ }^{5}$ (1996) e Sen et al. ${ }^{9}$ (1996).

A técnica de obturação empregada, o ângulo entre a parede dentinária e o túbulo, o diâmetro do

\section{REFERÊNCIAS}

1. Buchanan LS. The continuous wave of obturation technique: "centered" condensation of warm gutta-percha in 12 seconds. Dent Today 1996;15:60-7.

2. De Deus QD. Endodontia. $5^{\mathrm{a}}$ ed. Rio de Janeiro: Medsi; 1992.

3. Gutmann JL, Whitherspoon DE. Obturation of the cleaned and shaped root canal system. In: Cohen S, Burns RC. Pathways of the Pulp. $7^{\text {th }}$ ed. St. Louis: Mosby; 1998. p. 258-361.

4. Kouvas V, Liolios E, Vassiliadis L et al. Influence of smear layer on depth of penetration of three endodontic sealers: a SEM study. Endod Dent Traumatol 1998;14:191-5.

5. Leonard JE, Gutmann JL, Guo IY. Apical and coronal seal of roots obtured with dentine bonding agent and resin. Int Endod J 1996;29:76-83. túbulo e o tipo de cimento também são fatores que podem exercer influência na penetração dos cimentos nos túbulos dentinários ${ }^{4}$.

\section{CONCLUSÕES}

De acordo com os resultados obtidos com a metodologia empregada, podemos concluir que:

1. O cimento de Rickert (Pulp Canal Sealer) foi o que apresentou a maior capacidade de penetração intradentinária e o cimento de hidróxido de cálcio (Sealapex), a menor, sendo esta diferença estatisticamente significante.

2. A lama dentinária influenciou negativamente na capacidade de penetração intradentinária dos cimentos testados.

6. Oksan T, Aktener BO, Sen BH, Tezel H. The penetration of root canal sealers into dentinal tubules. A scanning electron microscope study. Int Endod J 1993;26:301-5.

7. Ruddle CJ. Three-dimensional obturation of the root canal system. Dent Today 1992;11:30-3,39.

8. Schilder H. Filling root canals in three dimensions. Dent Clin North Am 1967;11:723-44.

9. Sen BH, Piskin B, Baran N. The effect of tubular penetration of root canal sealers on dye microleakage. Int Endod $J$ 1996;29:23-8.

10. Siqueira Júnior FJ, Fraga RC, Garcia PF. Evaluation of sealing ability, $\mathrm{pH}$ and flow rate of three calcium hydroxide-based sealers. Endod Dent Traumatol 1995;11: 225-8.

11. Yared GM, Bou-Dagher F. Sealing ability of the vertical condensation with different root canal sealers. J Endod 1996;22:6-8.

12. Zidan O, Eldeeb ME. The use of a dentinal bonding agent as a root canal sealer. J Endod 1985;11:176-8.

Recebido para publicação em 11/03/02 Enviado para reformulação em 09/07/02 Aceito para publicação em 23/08/02 